\title{
Interaction of FOX-7 and Melatonin - A DFT Treatment
}

\section{Lemi Türker}

Department of Chemistry, Middle East Technical University, Üniversiteler, Eskişehir Yolu No: 1, 06800 Çankaya/Ankara, Turkey; e-mail: lturker@gmail.com; lturker@metu.edu.tr

\section{Abstract}

Within the limitations of density functional theory, the $\pi-\pi$ type interaction between melatonin (a nocturnal hormone) and FOX-7 (an insensitive explosive) is investigated. Two composite structures have been considered in which 6- and 5-membered aromatic rings of melatonin are $\pi-\pi$ linked to FOX-7 molecule. The structure optimization has been achieved at the level of B3LYP/6-31++G(d,p). The composite formed through the 5 -membered ring of melatonin is found more stable than the composite involving 6-membered ring of melatonin and both of them are more stable than the composite having no $\pi-\pi$ type interaction. Various structural, energetic, quantum chemical and spectral properties of the composites have been reported and discussed.

\section{Introduction}

Melatonin, a highly ubiquitous biomaterial, is secreted by pineal gland in brain. Although, some accumulated evidence exists indicating that melatonin is also synthesized in the hypothalamus and in other parts of the body, however the melatonin in the circulation originates from the pineal [1]. It has been found that its concentration in cerebrospinal fluid is higher than that in plasma [1]. It has been proved that the circulating melatonin level is interestingly dependent on the incident light, so that high levels are attained in darkness. Thus, melatonin is a nocturnal (sleep-related) hormone and inter-related with circadian rhythm, (known as circadian rhythm hormone) $[2,3]$.

Received: December 25, 2018; Accepted: January 6, 2019

Keywords and phrases: melatonin, FOX-7, DADNE, explosive, DFT.

Copyright (C) 2019 Lemi Türker. This is an open access article distributed under the Creative Commons Attribution License, which permits unrestricted use, distribution, and reproduction in any medium, provided the original work is properly cited. 


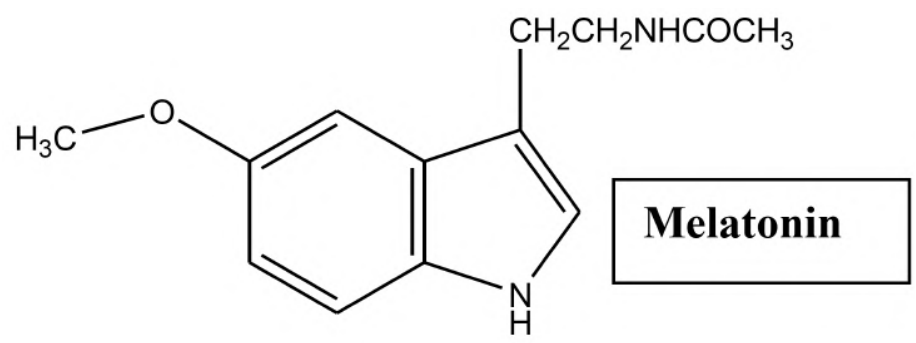

It has been clinically showed that melatonin treatment adversely affects the symptoms of rheumatoid arthritis [2]. A recent review article has reported some anticarcinogenic activity of melatonin [4]. It has been quite recently shown that of serum melatonin level determination is a valuable methodology to define body circadian phase in lung cancer patients [5]. It has been reported that melatonin reverses the darkening effect of melaninreleasing hormone $(\mathrm{MRH})$ by stimulating aggregation, causing lightening of skin color [6]. It has been also proved that melatonin exerts a certain retarding action on the esterus cycle. Since, melatonin also exists in hypothalamic tissue and peripheral nerve, it might possess some yet unknown functions in the metabolism of several neural and endocrine processes $[1,6,7]$. Considerable experimental evidence suggests that melatonin prevents accumulation of amyloid $\beta$ (A $\beta$ ) peptide in the brain which is supposed to be a cause of Alzheimer's disease [8]. Very recently, many articles piled up in the literature displaying its role in diverse fields of biology and medicine [9-17].

The melatonin biosynthesis originates from 5-hydroxy tryptophan which by decarboxylation in vivo produces serotonin (5-hydroxytryptamine). N-Acetylation of serotonin, followed by O-methylation in the pineal body forms melanotonin (melatonin, $\mathrm{N}$-acetyl-5-methoxyserotonin) [1,6]. Recent years also evidenced some physical and theoretical investigations on melatonin [18-22].

In the present study, interaction of melatonin and FOX-7 (diaminodinitroethylene, DADNE) has been considered within the constraints of density functional theory (DFT). Note that FOX-7 is a well known insensitive explosive [23] and attracted considerable attention in recent years [24]. It has good thermal stability and low sensitivity, exhibiting excellent application performance among the insensitive ammunitions and solid propellants. Although FOX-7 is simple in molecular composition and structure, its chemical reactivity is abundant and surprising, including salification reaction, coordination reactions, nucleophilic substitutions, acetylate reactions, oxidation and reduction reactions, electrophilic addition reactions $[25,26]$ etc. 


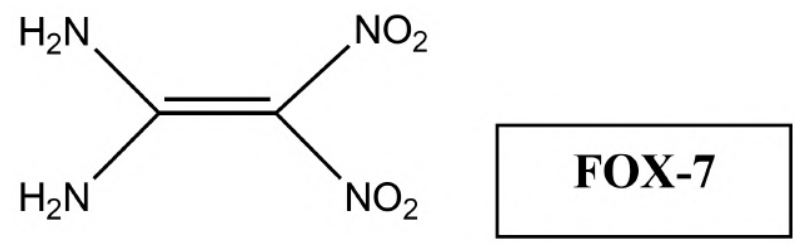

\section{Method of Calculation}

Presently, geometry optimizations of all the structures leading to energy minima were achieved first by using MM2 method [27, 28] for the equilibrium conformer. Subsequent optimizations were achieved at Hartree-Fock level using various basis sets hierarchically. Then, the geometry optimizations were managed within the framework of density functional theory $[29,30]$, finally at the levels of RB3LYP /6-31++G(d,p). Note that the exchange term of B3LYP consists of hybrid Hartree-Fock and local spin density (LSD) exchange functions with Becke's gradient correlation to LSD exchange [30, 31]. Note that the correlation term of B3LYP consists of the Vosko, Wilk, Nusair (VWN3) local correlation functional [32] and Lee, Yang, Parr (LYP) correlation correction functional [33]. The vibrational analyses have also been done. The total electronic energies are corrected for the zero point vibrational energy (ZPE). The stationary points to energy minima were proved in all the cases by calculation of the second derivatives of energy with respect to the atom coordinates. The normal mode analysis for each structure yielded no imaginary frequencies for the $3 N-6$ vibrational degrees of freedom, where $N$ is the number of atoms in the system. This indicates that the structure of each molecule corresponds to at least a local minimum on the potential energy surface. All these calculations were done by using the Spartan 06 package program [34].

\section{Results and Discussion}

Intermolecular forces, such as hydrogen bonds, dipole-dipole, charge-dipole and charge-charge interactions are responsible for various properties of matter. These same forces also lead to molecular recognition, specifically non-covalent association between a receptor molecule and a particular substrate. In some cases a special type of interaction, called $\pi-\pi$ interaction occurs, especially if the interacting molecules are donor-acceptor type. Most of the explosive molecules, due to strong electron acceptor groups (such as nitro groups) present in their structures behave as acceptor molecules. 
FOX-7 molecule is of considerable interest in terms of $\pi-\pi$ interactions because it possesses both the acceptor groups $\left(\mathrm{NO}_{2}\right)$ and the donor groups $\left(\mathrm{NH}_{2}\right)$, namely it should be ambidient. The molecule itself is a push-pull type structure [35]. FOX-7 can be represented by various resonance forms (I, II, etc.) and various 1,5-type proton tautomers [35, 36] (III, etc).

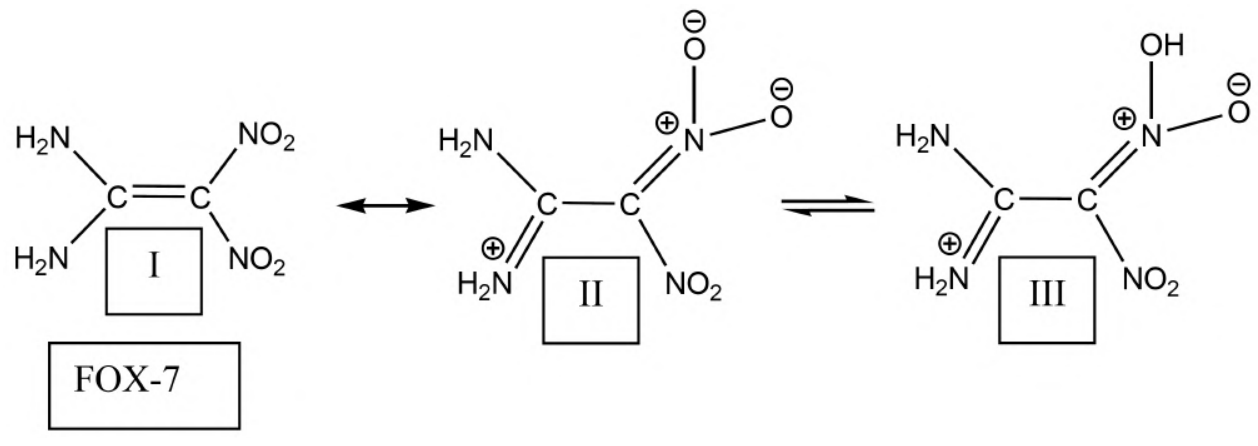

The $\pi$-stacking interactions are sometimes possible in which the planar $\pi$-systems of aromatic molecules or aromatic macro cycles lay one on top of the other with nearly parallel orientation (Figure 1). Such interactions are responsible for the stacking of hydrogen-bonded base pairs in DNA [37].

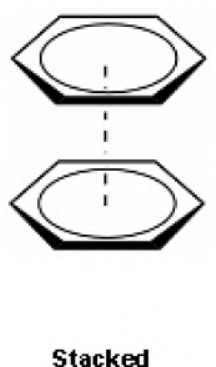

Stacked

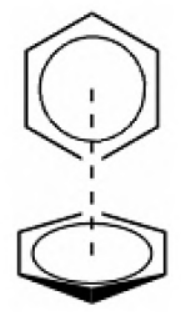

T-shaped
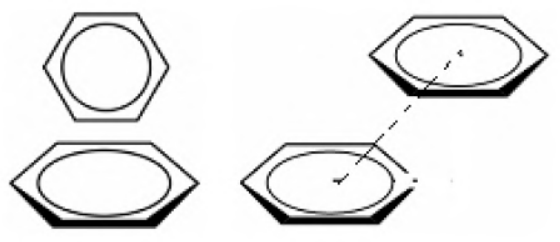

Edgeto-face

Displaced or stip stacked

Figure 1. Types of $\pi-\pi$ stacking interactions.

Although, the term, aromatic-aromatic interactions, imply stacking of two $\pi$-systems on top of each other, it is not always the case. For instance, two benzene molecules orient themselves perpendicular to each other. To directly stack two benzenes on top of one another leads to an adverse repulsion of electrostatic origin [38]. Nevertheless, simple aromatics do have favorable interactions with each other. Systems like benzene prefer T-shaped or edge-to-face interaction better than stacking. In some more complicated structures, it is best to form a displaced or slipped stack orientation (Figure 1). 
This slipped stack orientation still aligns regions of positive electrostatic potential with regions of negative electrostatic potential which leads to energetically favorable system ( $\pi$-staking). $\pi$-donor-acceptor interaction occurs between any two molecules or regions of a molecule where one has a low energy empty orbital (acceptor) and the other a high energy filled orbital (donor). When these two orbitals have proper orientation in space, some extent of charge transfer can occur from the donor to the acceptor molecule [38]. Note that the methoxy group substituted to 6-membered ring of melatonin should make that ring mesomerically electron rich. The methylene group substituted to the 5-membered ring inductively activates the ring towards electron acceptor molecules. Melatonin and FOX-7 molecules in the vicinity of each other are under the influence of various forces. The $\pi-\pi$ interaction is one of them which is interesting to contemplate on it.
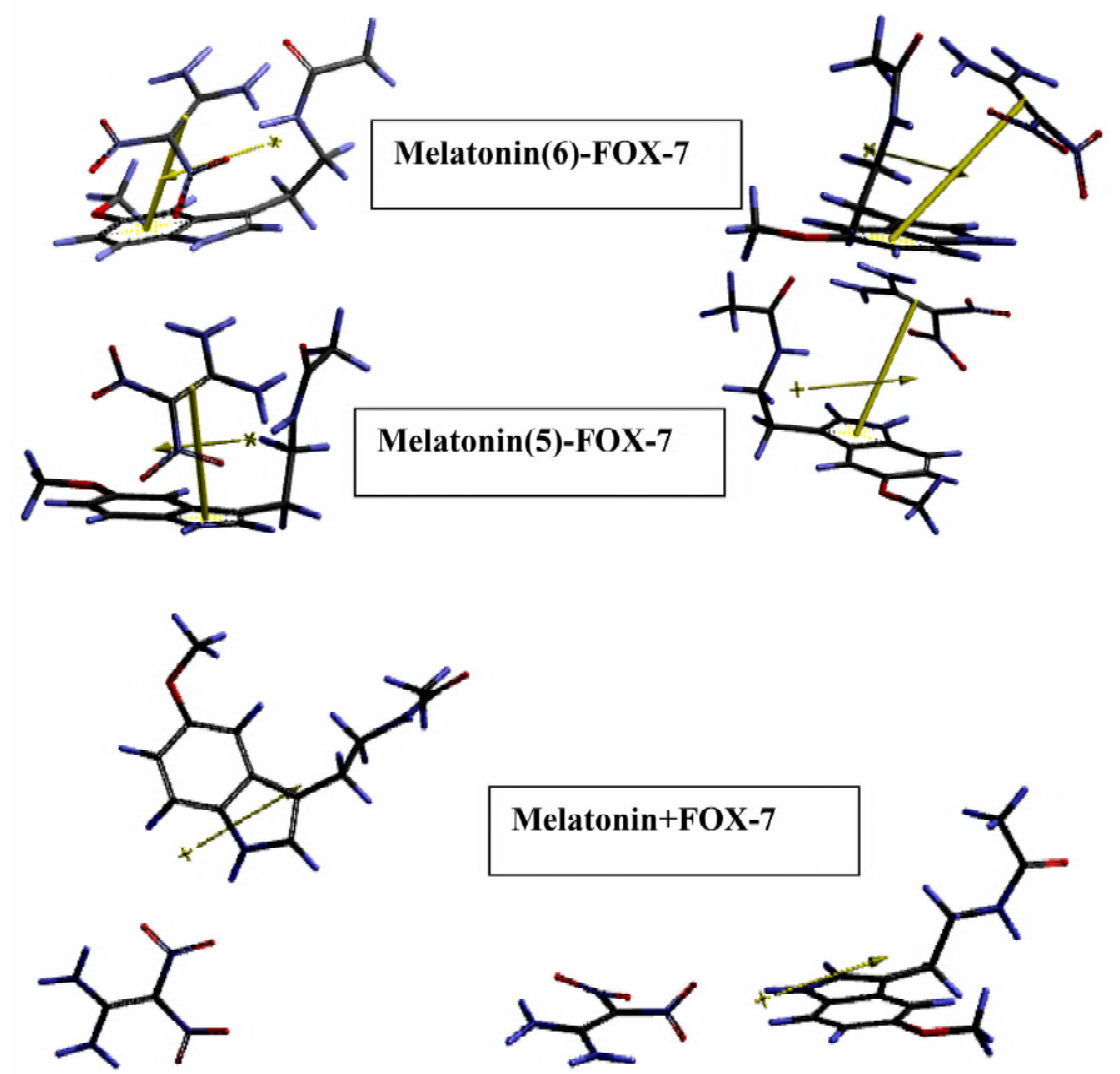

Figure 2. Optimized structures of the composites considered (from different angles of view). 
Figure 2 shows the optimized structures of the composites of melatonin and FOX-7. Note that $\pi-\pi$ interaction of FOX-7 with melatonin might be through the 6- or 5 -membered ring of melatonin which are indicated by melatonin(6)-FOX-7 and melatonin(5)-FOX-7, respectively. As seen from the figure the assumed $\pi-\pi$ interaction is slip-stacked type. On the other hand, melatonin+ FOX-7 stands for the composite without any $\pi-\pi$ interactions. Note that the amino groups of FOX-7 is next to the amide carbonyl of melatonin, indicating that some charge-charge or/and dipole-dipole etc., type interactions also should be present together with the pre-assumed $\pi-\pi$ interactions.
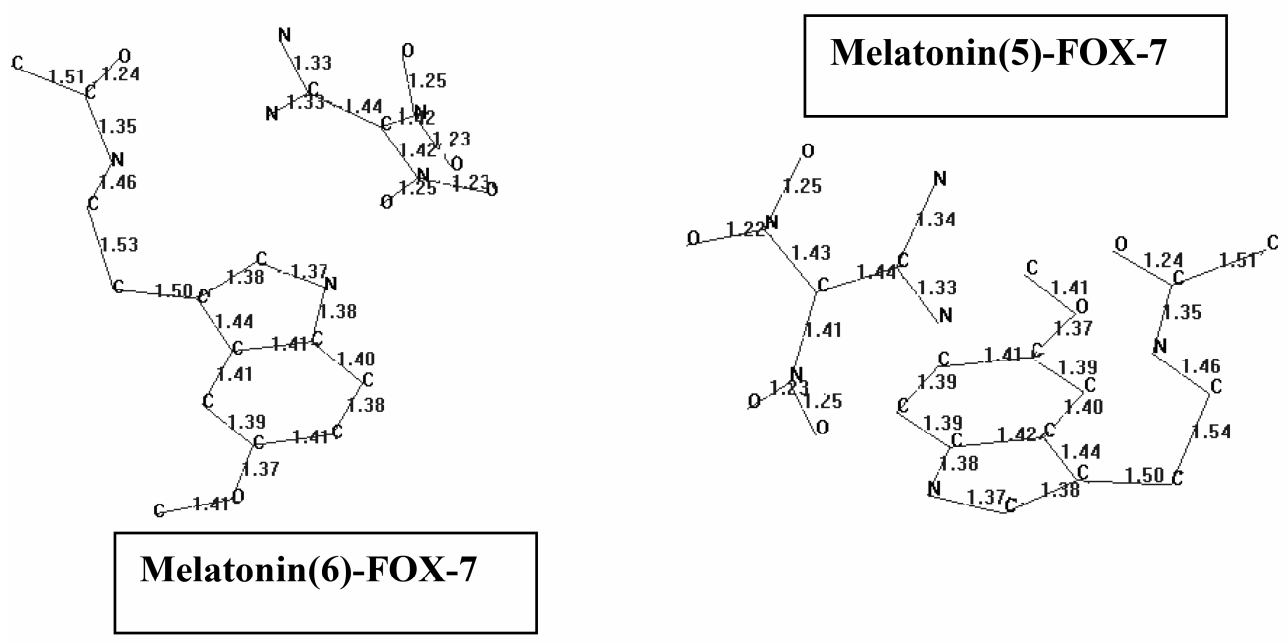

Figure 3. Bond lengths ( $\AA$ ) in the $\pi$ - $\pi$-interacted composite structures considered (Hydrogens omitted).

Figure 3 shows the bond lengths in the $\pi$ - $\pi$-interacted composites considered which indicate no appreciable changes in the lengths of respective bonds. The distance/bond standing for the $\pi$ - $\pi$-interaction between the stacks are $5.14 \AA$ and $4.48 \AA$ for melatonin(6)-FOX-7 and melatonin(5)-FOX-7, respectively. Table 1 displays some properties of the $\pi$ - $\pi$-interacted composites which are very comparable for both of the composites. Figure 4 shows the electrostatic charges (ESP) on the $\pi$ - $\pi$-interacted structures considered. Note that the ESP charges are obtained by the program based on a numerical method that generates charges that reproduce the electrostatic potential field from the entire wave function [34]. 
Table 1. Various QSAR properties of the $\pi$ - $\pi$-interacted composites considered.

\begin{tabular}{lcc}
\hline Property & Melatonin(6)-FOX-7 & Melatonin(5)-FOX-7 \\
\hline Area $\left(\AA^{2}\right)$ & 397.38 & 398.98 \\
Volume $\left(\AA^{3}\right)$ & 355.75 & 355.96 \\
Ovality $(\mathrm{CPK})$ & 1.64 & 1.64 \\
Polarizability & 69.54 & 69.56 \\
Log P & 0.14 & -0.40 \\
\hline
\end{tabular}
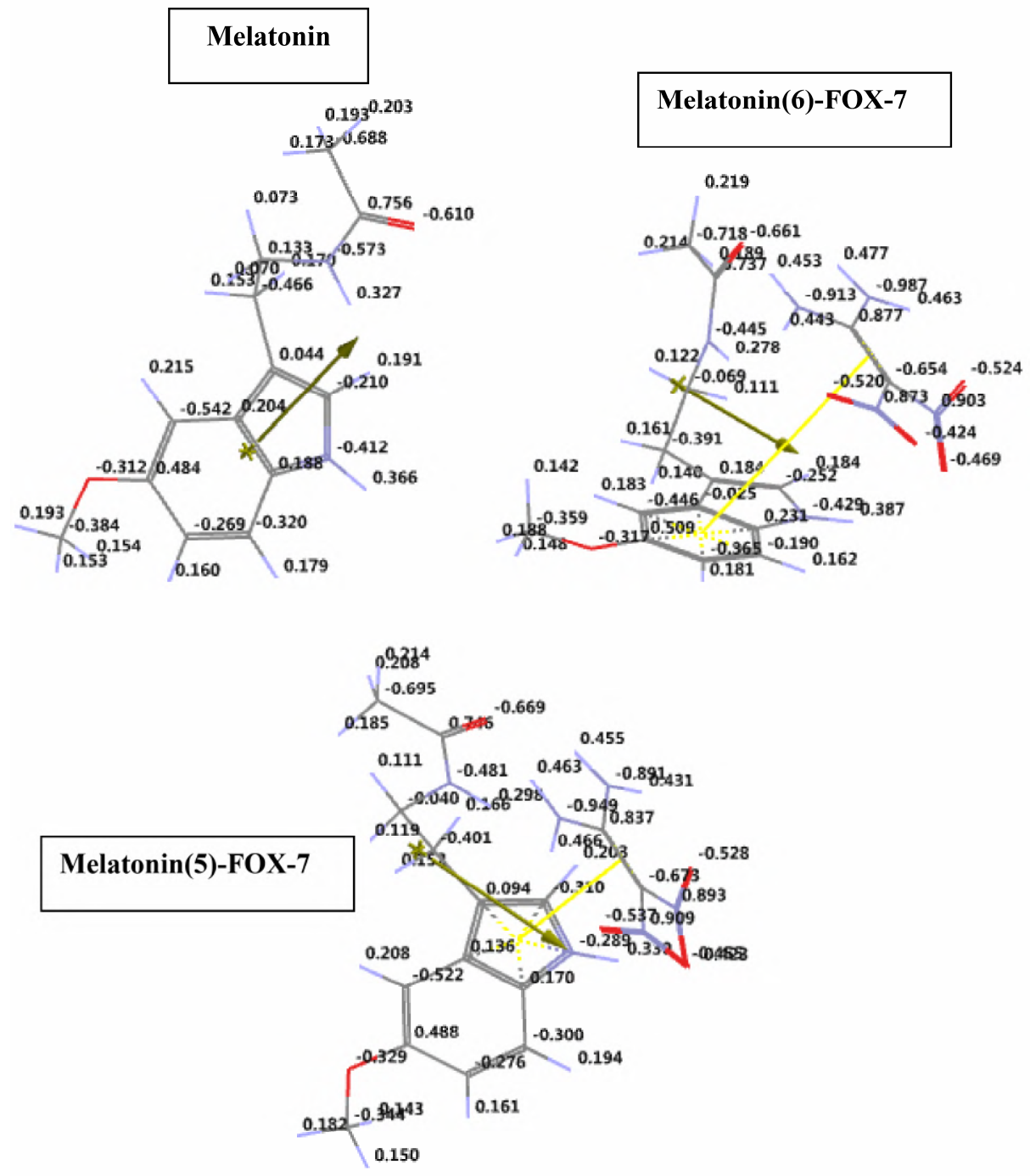

Figure 4. Electrostatic (ESP) charges on the $\pi$ - $\pi$-interacted systems considered. 
Various energies of the composites are shown in Table 2 where E, ZPE and $\mathrm{E}_{\mathrm{c}}$ stand for the total electronic energy, zero point vibrational energy and corrected total energy, respectively. As seen there, the composite formed through the 5-membered ring of melatonin is comparatively more stable. Note that the $\pi$-bond between the stacks in that case is shorter (4.48 $\AA$ ) compared to interaction through the 6-membered ring (5.14 $)$ ). Although, the 6-membered ring is expected to be more electron rich due to the mesomeric effect of methoxy oxygen atom, the more favorable energy of composite having interaction through 5-memebered ring might be due to better overlap or interaction of orbitals in that case.

Table 2. Various energies of the composite systems considered.

\begin{tabular}{lccc}
\hline Systems & $\mathbf{E}$ & ZPE & $\mathbf{E}_{\mathbf{c}}$ \\
\hline Melatonin(6)-FOX-7 & -3579648.04 & 967.28 & -3578680.76 \\
Melatonin(5)-FOX-7 & -3579657.08 & 966.97 & -3578690.11 \\
\hline Melatonin+ FOX-7 & -3579606.83 & 962.14 & -3578644.69 \\
\hline
\end{tabular}

Energies in $\mathrm{kJ} / \mathrm{mol}$. RB3LYP/6-31++G(D,P)

Figure 5 shows the IR spectra of the composites considered. In the case of melatonin(6)-FOX-7 composite, the asymmetric N-H stretchings of FOX-7 moiety occur at $3630-3620 \mathrm{~cm}^{-1}$, followed by N-H stretching of the 5-membered ring $\left(3593 \mathrm{~cm}^{-1}\right)$. The amide N-H stretching occurs at $3545 \mathrm{~cm}^{-1}$. The vibrations around $3493 \mathrm{~cm}^{-1}$ stand for the symmetrical $\mathrm{N}-\mathrm{H}$ stretchings of the FOX-7 moiety. The amide $\mathrm{C}=\mathrm{O}$ stretching happens at $1694 \mathrm{~cm}^{-1}$. The asymmetrical $\mathrm{N}-\mathrm{O}$ stretchings of $\mathrm{NO}_{2}$ groups are coupled with N-H bendings of FOX-7 component. In the case of melatonin(5)-FOX-7 composite, the asymmetric N-H stretchings of FOX-7 moiety occur at $3648 \mathrm{~cm}^{-1}$. The vibration at $3628 \mathrm{~cm}^{-1}$ is for the ring N-H stretching of pyrrole moiety. The asymmetric N-H stretchings of FOX-7 moiety occur at $3598 \mathrm{~cm}^{-1}$. The amide N-H stretching occurs at $3543 \mathrm{~cm}^{-1}$. The symmetrical N-H stretchings of FOX-7 component occur about 3393 $\mathrm{cm}^{-1}$. The amide $\mathrm{C}=\mathrm{O}$ stretching is observed at $1695 \mathrm{~cm}^{-1}$. As for the melatonin+FOX-7 composite (having no $\pi-\pi$ interaction) $3596 \mathrm{~cm}^{-1}$ stands for amide N-H stretching of melatonin. $3695 \mathrm{~cm}^{-1}$ and $3474 \mathrm{~cm}^{-1}$ are for the asymmetrical N-H stretchings of FOX-7 component. The carbonyl stretching occurs at $1743 \mathrm{~cm}^{-1}$. The asymmetrical N-O stretching of $\mathrm{NO}_{2}$ groups happens at $1605 \mathrm{~cm}^{-1}$. 

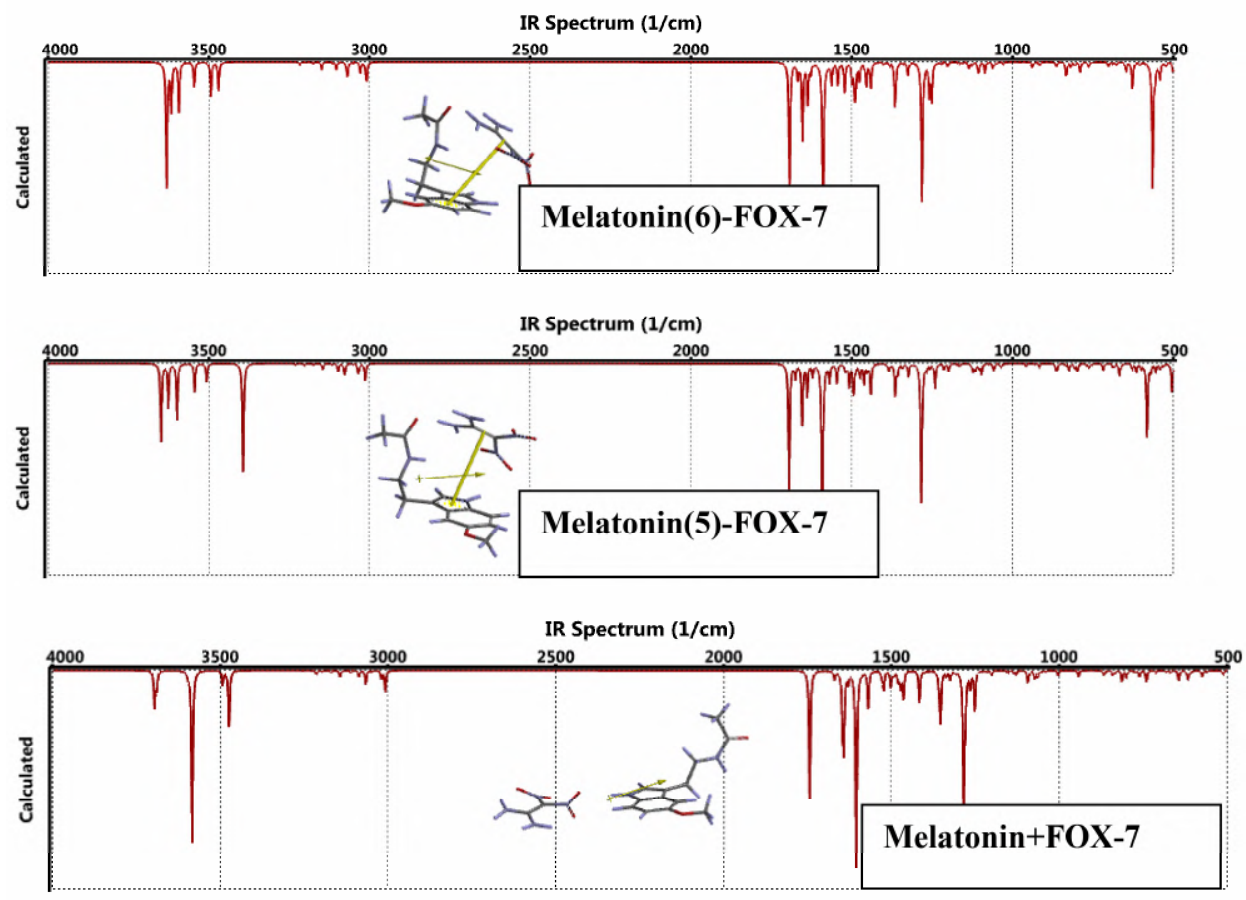

Figure 5. Calculated IR spectra of the composites considered.

The calculated time-dependent UV-VIS spectra of the composites considered are shown in Figure 6 . The two spectra of $\pi$ - $\pi$-interacted composites differ partly in the UV and mainly in the visible regions. Melatonin(5)-FOX-7 composite is characterized with comparatively lower absorptions in the visible part indicating that $\pi-\pi$ interaction between the components of the composite affects the likely hood of transitions occurring in the visible region. As for melatonin+FOX-7 composite the spectrum mainly covers the visible region. In this composite interactions between the components should be the set of charge-charge, charge dipole or/and dipole-dipole type and they perturb the molecular orbitals of the system very different than the composites in which also $\pi$ - $\pi$-interaction takes place beside the other types of interactions occurring in them. 

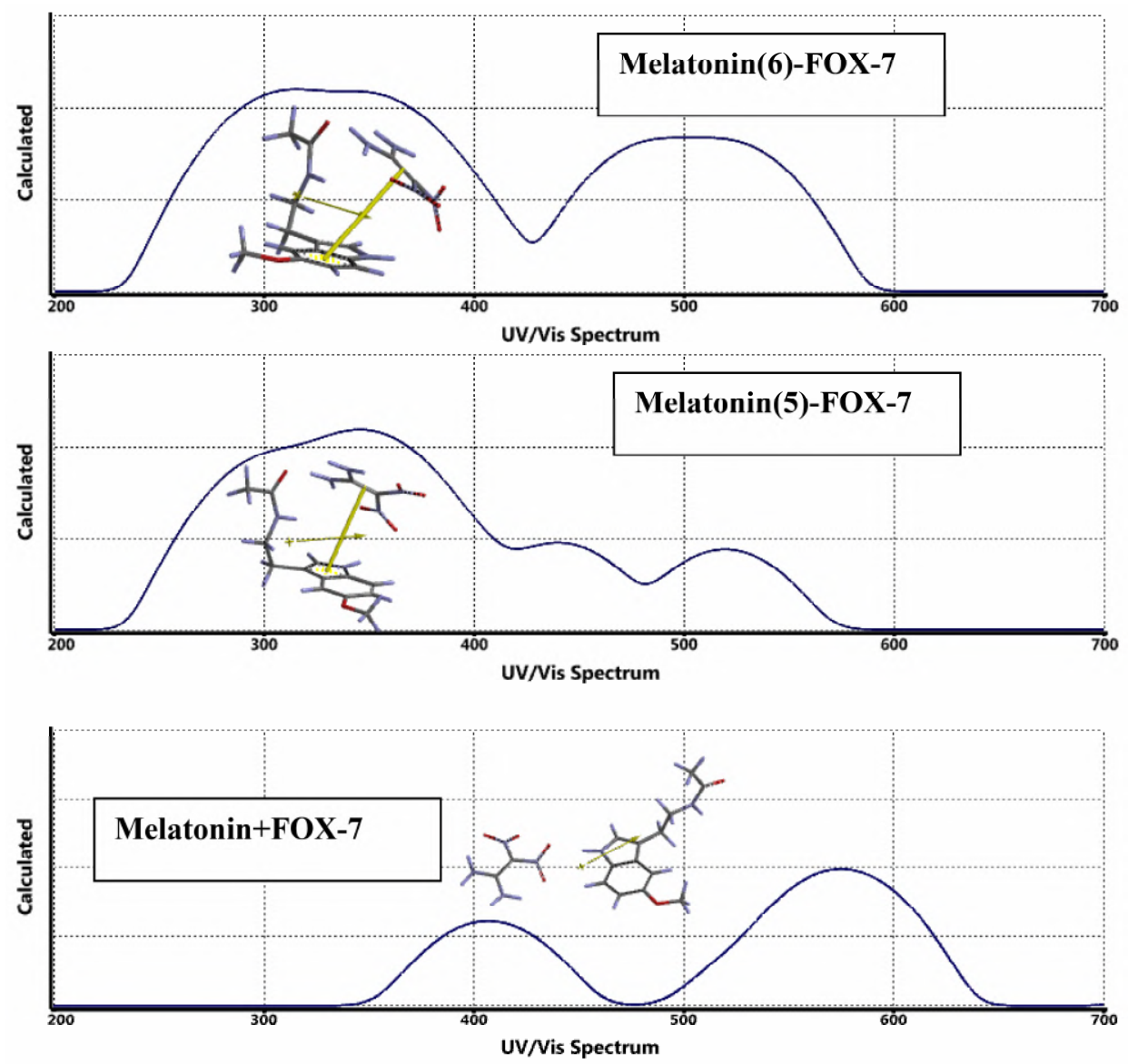

Figure 6. Calculated UV-VIS spectra of the composites considered.

Table 3 shows the HOMO, LUMO energies and interfrontier molecular orbital gaps $(\Delta \varepsilon)$ of the composite systems considered. As seen in the table the HOMO and LUMO energies of melatonin(6)-FOX-7 composite are lower (more negative values) than the respective orbitals of the other composite which indicate that the mesomeric effect of methoxy oxygen is not so effective to raise the HOMO and LUMO energies as expected. As for the interfrontier molecular orbital gaps $(\Delta \varepsilon)$ melatonin(6)-FOX-7 has slightly narrower gap as compared to the other composite. Note that melatonin+ FOX-7 composite possesses the highest HOMO and lowest LUMO energies among the composites. 
Table 3. The HOMO, LUMO energies and interfrontier molecular orbital gaps $(\Delta \varepsilon)$ of the systems considered.

\begin{tabular}{lccc}
\hline Systems & HOMO & LUMO & $\boldsymbol{\Delta} \boldsymbol{\varepsilon}$ \\
\hline Melatonin & -541.60 & -80.67 & 460.93 \\
FOX-7 & -724.89 & -276.96 & 447.93 \\
Melatonin(6)-FOX-7 & -549.00 & -266.76 & 282.24 \\
Melatonin(5)-FOX-7 & -543.53 & -261.04 & 282.49 \\
\hline Melatonin+ FOX-7 & -512.94 & -326.93 & 186.01 \\
\hline
\end{tabular}

Energies in kJ/mol. RB3LYP/6-31++G(D,P).

Figures 7 and 8 are each time one component-deleted (decomposed composite) UVVIS spectra of the $\pi$ - $\pi$-interacted composites such that geometry of the rest has been kept as in the full composite structure. To obtain the spectra of the remnant single point calculations has been done using the same calculation level of DFT.

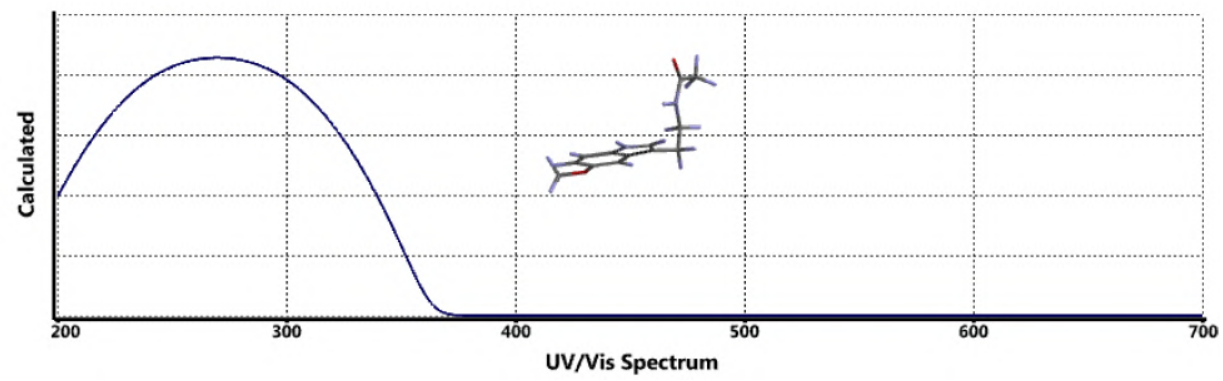

Figure 7. Calculated UV-VIS spectra of the composite (Melatonin(5)-FOX-7) after FOX-7 structure is deleted.

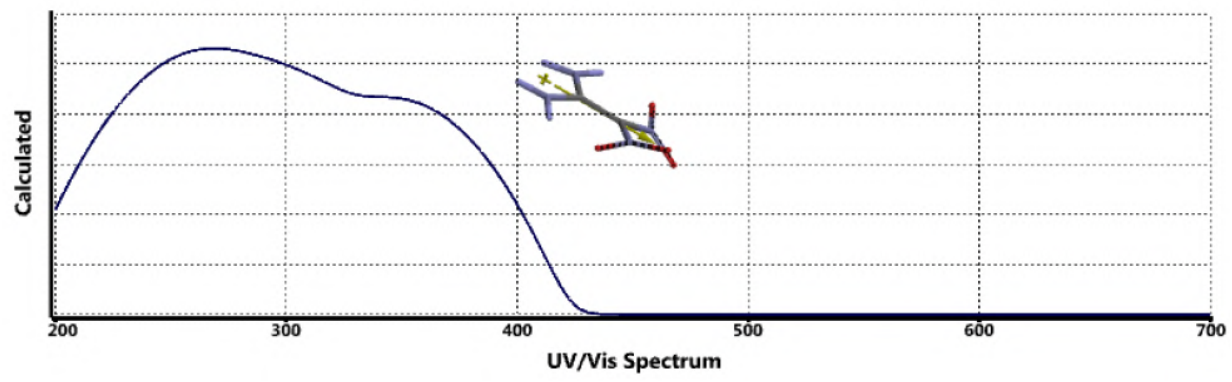

Figure 8. Calculated UV-VIS spectra of the composite (Melatonin(5)-FOX-7) after melatonin structure is deleted. 
As seen in Figures 7 and 8, both of the components absorbs in the UV region and compared to Figure 6, the effect of overall interaction between the components is very dramatic. However, the $\pi-\pi$ interaction between FOX-7 and either through 6- or 5 -membered rings of melatonin results the emergence of bathochroming effect in the spectra of composites. Thus, in the composites considered some sort of $\pi-\pi$ interaction exists.

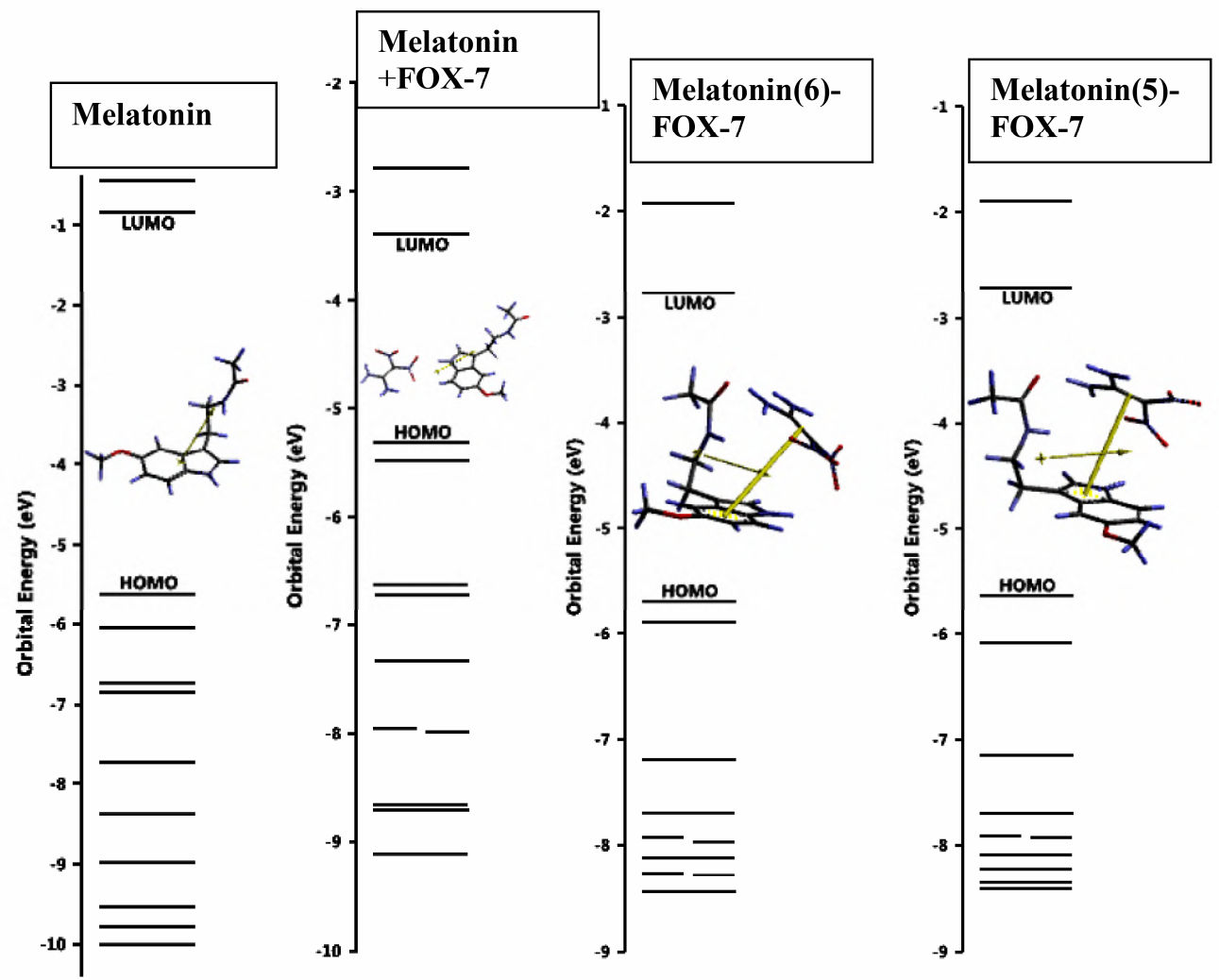

Figure 9. Some of the molecular orbital energies of melatonin and the composites considered.

Figure 9 shows some of the molecular orbital energy levels of melatonin and the composites considered. The energy spectra of both of the $\pi$ - $\pi$-interacted composites are very similar, however the NEXTHOMO energy level of melatonin(5)-FOX-7 composite is lower compared to the respective level of the other composite. On the other hand, it is clearly seen that the presence of FOX-7 highly perturbs the molecular orbital energy levels of melatonin. 


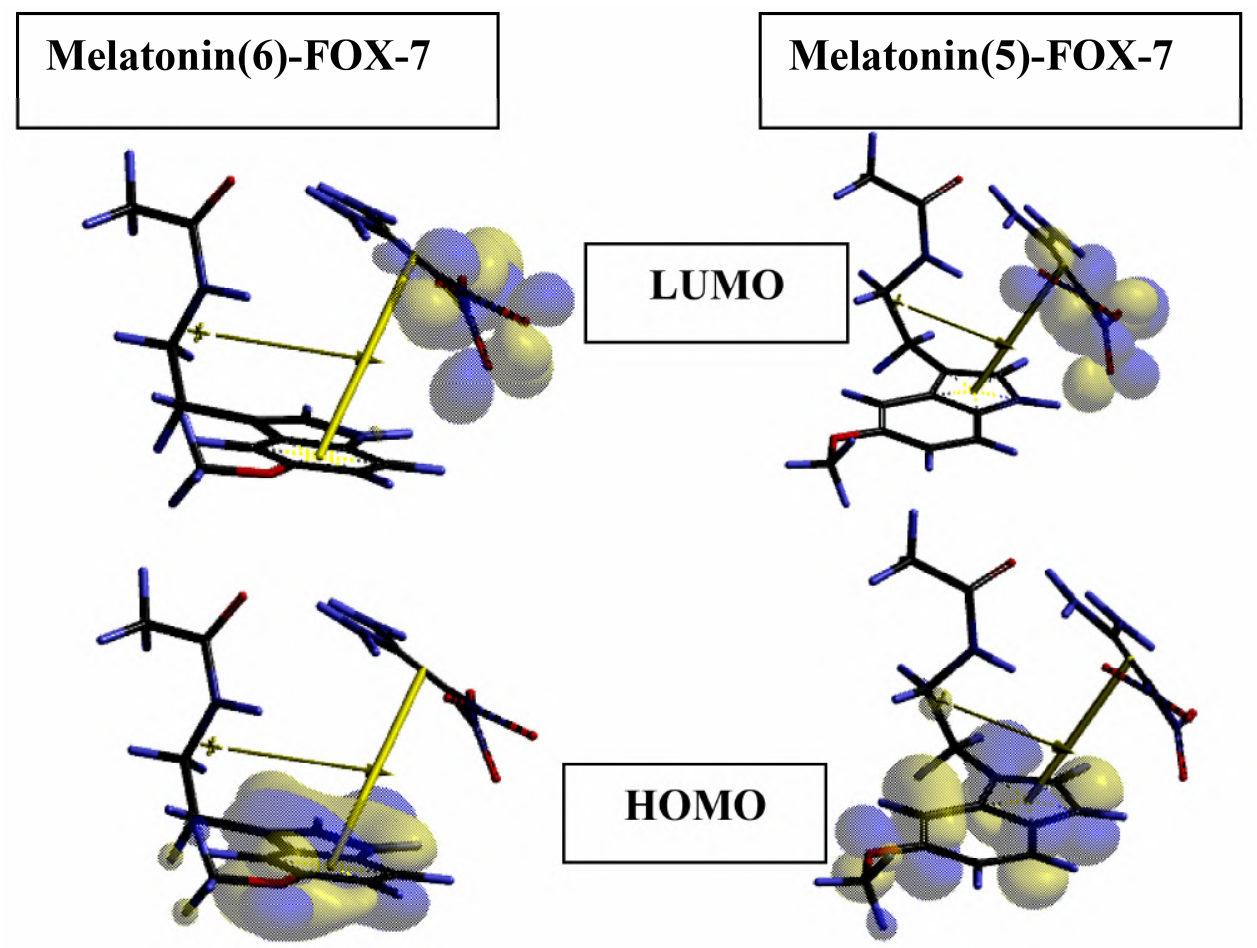

Figure 10. The HOMO and LUMO pattern of the $\pi$ - $\pi$-interacted composites.

Figure 10 shows the HOMO and LUMO patterns of the $\pi$ - $\pi$-interacted composites. As seen in the figure, in both cases of interaction, the HOMO is confined to aromatic part of melatonin component whereas the LUMO spreads over the nitro groups of FOX-7 component.
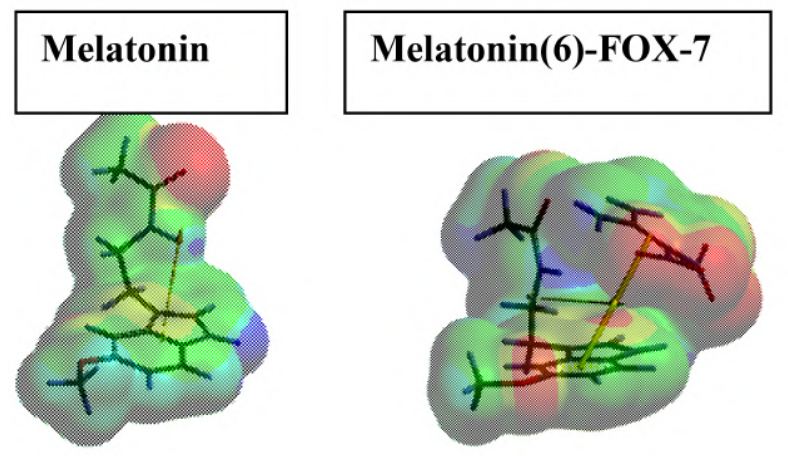

\section{Melatonin(5)-FOX-7}

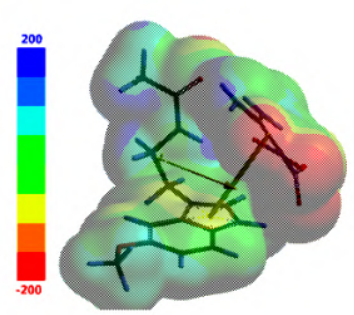

Figure 11. Electrostatic potential maps of melatonin and the $\pi$ - $\pi$-interacted composites considered. 
Electrostatic potential maps of the composites considered are shown in Figure 11. In melatonin negative electrostatic potential region is over the amide carbonyl group (red region). In the composites, FOX-7 nitro groups are characterized with negative electrostatic potential. However, in the case of melatonin(6)-FOX-7 composite some negative electrostatic potential region appears on the methoxy substituent of melatonin. The melatonin backbone is superimposed with various degrees of electropositive potential (blue/green regions). Also note that, in the composites the amide carbonyl of melatonin component no longer possesses a negative potential.

\section{Conclusion}

The present DFT treatment has indicated that melatonin and FOX-7 interact strongly via various interaction types. One of them is the presently focused $\pi$ - $\pi$ type interaction which occurs in the form of slip-stack orientation and more favorable (although entropically disfavored) than the one without $\pi-\pi$ interaction. Of the two possible $\pi-\pi$ type interacted composites, the one formed by the involvement of 5-membered ring of melatonin seems to be more favorable over the interaction through the 6-membered ring. Although FOX-7 is a push-pull type $\pi$-system, it has still some potential to link itself to melatonin which is an electron rich system. As a result of the mutual interaction, various properties of melatonin have been perturbed which might induce some toxic effects.

\section{References}

[1] F. S. Greenspan and P. H. Forsham, Basic and Clinical Endocrinology, Drawer L, Los Altos, California: Lange Medical Pub., 1983, p.33.

[2] M. Cutolo, R. Straub and F. Buttgereit, Circadian rhythms of nocturnal hormones in rheumatoid arthritis: translation from bench to bedside, Annals of the Rheumatic Diseases 67 (2008), 905-908.

[3] H. Tagaya, Measurement of sleep-related hormones, Biomedical Engineering 46(2) (2008), 169-176.

[4] A. Bukowska, Anticarcinogenic role of melatonin-potential mechanisms, Medycyna Pracy 62 (2011), 425-434.

[5] G. Mazzoccoli, F. Giuliani and R. B. Sothern, Determination of whole body circadian phase in lung cancer patients: melatonin vs. cortisol, Cancer Epidemiology 36 (2012), 4653. 
[6] A. W. White, P. Handler and E. L. Smith, Principles of Biochemistry, New York: McGraw-Hill, 1968, p. 985.

[7] R. K. Murray, D. K. Granner, P. A. Mayes and V. W. Rodwell, Harper's Biochemistry, East Norwalk, Connecticut: Lange Medical Pub., 1988.

[8] C. J. Pike, D. Burdick, A. J. Walencewitz, C. G. Glabe and C. W. Cotman, Neurodegeneration induced by $\beta$-amyloid peptides in vitro: the role of peptide assembly state, J. Neurosci. 13 (1993), 1676-1687.

[9] H. M. Foley and A. E. Steel, Adverse events associated with oral administration of melatonin: A critical systematic review of clinical evidence, Complementary Therapies in Medicine 42 (2019), 65-81.

[10] Z. Xu, Y. Wu, Y. Zhang, H. Zhang and L. Shi, Melatonin activates BKCa channels in cerebral artery myocytes via both direct and MT receptor/PKC-mediated pathway, European Journal of Pharmacology 842 (2019), 177-188.

[11] C. Luo, Q. Yang, Y. Liu, S. Zhou, J. Jiang, R. J. Reiter, P. Bhattacharya, Y. Cui, H. Yang, H. Ma, J. Yao, S. E. Lawler, X. Zhang, J. Fu, R. Rozental, H. Aly, M. D. Johnson, E. A. Chiocca and $\mathrm{X}$. Wang, The multiple protective roles and molecular mechanisms of melatonin and its precursor $\mathrm{N}$-acetylserotonin in targeting brain injury and liver damage and in maintaining bone health, Free Radical Biology and Medicine 130 (2019), 215-233.

[12] A. Rafat, A. M. Roushandeh, A. Alizadeh, N. Hashemi-Firouzi and Z. Golipoor, Comparison of the melatonin preconditioning efficacy between bone marrow and adiposederived mesenchymal stem cells, Cell Journal 20(4) (2019), 450-458.

[13] S. Mukherjee, Recent advancements in the mechanism of nitric oxide signaling associated with hydrogen sulfide and melatonin crosstalk during ethylene-induced fruit ripening in plants, Nitric Oxide - Biology and Chemistry 82 (2019), 25-34.

[14] C. N. Campos, R. G. Ávila, K. R. D. de Souza, L. M. Azevedo and J. D. Alves, Melatonin reduces oxidative stress and promotes drought tolerance in young Coffea arabica L. plants, Agricultural Water Management 211 (2019), 37-47.

[15] J. A. Abecia, F. Forcada, M. I. Vázquez, T. M. Blanco, J. A. Pérez-Ce, R. Pérez-Pe and A. Casao, Role of melatonin on embryo viability in sheep, Reproduction, Fertility and Development 31(1) (2019), 82-92.

[16] A. Hosseinzadeh, S. A. Javad-Moosavi, R. J. Reiter, R. Yarahmadi, H. Ghaznavi and S. Mehrzadi, Oxidative/nitrosative stress, autophagy and apoptosis as therapeutic targets of melatonin in idiopathic pulmonary fibrosis, Expert Opinion on Therapeutic Targets 22(12) (2018), 1049-1061. 
[17] T. Y. Kuznetsova, N. V. Solovyova, V. V. Solovyov and V. O. Kostenko, Antioxidant activity of melatonin and glutathione interacting with hydroxyl and superoxide anion radicals, Ukrainian Biochemical Journal 89(6) (2017), 22-30.

[18] S. A. Pshenichnyuk, A. Modelli, D. Jones, E. F. Lazneva and A. S. Komolov, Low-energy electron interaction with melatonin and related compounds, Journal of Physical Chemistry B 121(16) (2017), 3965-3974.

[19] M. Kubota and T. Kobayashi, Electronic structures of melatonin and related compounds studied by photoelectron spectroscopy, Journal of Electron Spectroscopy and Related Phenomena 128(2-3) (2003), 165-178.

[20] D. Vasilescu and H. Broch, Quantum molecular modeling of melatonin, Journal of Molecular Structure: Theochem 460(1-3) (1999), 191-205.

[21] D. F. Lewis, J. Arendt and J. English, Quantitative structure-activity relationships within a series of melatonin analogs and related indolealkylamines. Journal of Pharmacology and Experimental Therapeutics 252(1) (1990), 370-373.

[22] L. Türker and T. Atalar, Interaction between TNT and melatonin-DFT treatment, Polycyclic Aromatic Compounds 32 (2012), 615-625.

[23] J. P. Agrawal, High Energy Materials, Weinheim: Wiley-VCH, 2010.

[24] W. A. Trzciński and A. Belaada, 1,1-Diamino-2,2-dinitroethene (DADNE, FOX-7) Properties and formulations (a Review), Central European Journal of Energetic Materials (CEJEM) 13(2) (2016), 527-544.

[25] Y. Zhang, Q. Sun, K. Xu, J. Song, F. Zhao, Review on the reactivity of 1,1-diamino-2,2dinitroethylene (FOX-7), Propellants, Explos., Pyrotech. 41 (2016), 35-52.

[26] K. Baum, N.V. Nguyen, R. Gilardi, J. L. Flippen-Anderson and C. George, Nitration of 1,1-diamino-2,2-dinitroethylenes, Journal of Organic Chemistry 57 (1992), 3026-3030.

[27] A. R. Leach, Molecular Modeling, Essex: Longman, 1997.

[28] P. Fletcher, Practical Methods of Optimization, New York: Wiley, 1990.

[29] W. Kohn and L. Sham, Self-consistent equations including exchange and correlation effects, J. Phys. Rev. 140 (1965), 1133-1138.

[30] R. G. Parr and W. Yang, Density Functional Theory of Atoms and Molecules, London: Oxford University Press, 1989.

[31] A. D. Becke, Density-functional exchange-energy approximation with correct asymptotic behavior, Phys. Rev. A 38 (1988), 3098-3100. 
[32] S. H. Vosko, L. Vilk and M. Nusair, Accurate spin-dependent electron liquid correlation energies for local spin density calculations: a critical analysis, Can. J. Phys. 58 (1980), 1200-1211.

[33] C. Lee, W. Yang and R. G. Parr, Development of the Colle-Salvetti correlation-energy formula into a functional of the electron density, Phys. Rev. B 37 (1988), 785-789.

[34] SPARTAN 06, Wavefunction Inc., Irvine CA, USA, 2006.

[35] L. N. Ferguson, The Modern Structural Theory of Organic Chemistry, New Delhi: Prentice-Hall, 1969.

[36] O. Reutov, Theoretical Principles of Organic Chemistry, Moscow: Mir, 1970.

[37] P. Atkins and J. de Paula, Atkins' Physical Chemistry, 7th ed., New York, USA: Oxford University Press, 2002, pp. 697-698.

[38] E. V. Anslyn and D. A. Dougherty, Modern Physical Organic Chemistry, Sausalito, California, USA: University Science Books, 2006, p. 184, 186. 